Research Paper

\title{
Aldo-Keto Reductase Family 1 Member B10 (AKR1B10) overexpression in tumors predicts worse overall survival in hepatocellular carcinoma
}

\author{
Jia Shi ${ }^{*}$, Lixiang Chen ${ }^{*}$, Yi Chen ${ }^{3 *}$, Yunfei Lu1 ${ }^{1}$, Xiaorong Chen ${ }^{1}$, Zongguo Yang1 ${ }^{\circledR}$ \\ 1. Department of Integrative Medicine, Shanghai Public Health Clinical Center, Fudan University, Shanghai 201508, China. \\ 2. Department of Laboratory Animal, Shanghai Public Health Clinical Center, Fudan University, Shanghai 201508, China. \\ 3. Department of Hepatobiliary Surgery, Shanghai Public Health Clinical Center, Fudan University, Shanghai 201508, China. \\ * These authors have contributed equally to this work. \\ $\square$ Corresponding author: Zongguo Yang, MD, PhD, Shanghai Public Health Clinical Center, Fudan University, Shanghai 201508, China; Email: \\ yangzongguo@shphc.org.cn \\ (c) The author(s). This is an open access article distributed under the terms of the Creative Commons Attribution License (https://creativecommons.org/licenses/by/4.0/). \\ See http://ivyspring.com/terms for full terms and conditions.
}

Received: 2019.01.03; Accepted: 2019.07.28; Published: 2019.08.27

\begin{abstract}
Overexpression of AKR 1B 10 correlated with tumorigenesis of many human malignancies; however, the prognostic value of AKR IB 10 expression in patients with hepatocellular carcinoma $(\mathrm{HCC})$ still remains controversial. In this analysis, AKR1B10 expression in HCC tumors were evaluated in GEO, TCGA and Oncomine databases, and a survival analysis of AKRIB10 based on TCGA profile was performed. We found that AKR $1 \mathrm{~B} 10$ was significantly overexpressed in tumors compared with nontumors in 7 GEO series (GSE14520, GSE25097, GSE33006, GSE45436, GSE55092, GSE60502, GSE77314) and TCGA profile (all $P<0.05$ ). Meta-analysis in Oncomine database revealed that AKRIB10 was significantly upregulated in cirrhosis, liver cell dysplasia and HCC compared with normal tissues (all $P<0.05$ ). Kaplan-Meier analysis demonstrated that high AKR1B10 in tumors were significantly associated with worse overall survival $(O S)$ in HCC patients $(P<0.05)$. Subgroup analysis showed that AKR 1B10 overexpression were associated with poor 1-year, 3-year and 5-year OS (all $P<0.05$ ). In addition, prognostic values of AKR1B10 upregulation for OS were more significant in HCC with hepatitis-virus-free $(P=0.00055)$, White race $(P=0.0029)$ and alcohol-free $(P=0.013)$, and both in male and female $(P=0.014$ and $P=0.034$, respectively). In conclusion: $A K R 1 B 10$ was upregulated in tumors and correlated with worse OS in HCC patients.
\end{abstract}

Key words: AKR1B10; hepatocellular carcinoma; survival; outcome

\section{Introduction}

Liver cancer, comprising $75 \% \sim 85 \%$ cases of hepatocellular carcinoma (HCC), is predicted to be the sixth most commonly diagnosed cancer and the fourth leading cancer related deaths worldwide in 2018 [1-4]. In addition, the incidence of HCC will continue to rise over the next 10-20 years and to peak around 2030 based on a SEER registry projects study $[3,5]$. There has been a marked increase in HCC related annual death rates during the past two decades [3]. Precise estimation of prognosis plays a critical role in treatment decision in HCC patients.
Thus, to identify reliable prognostic biomarkers and to reveal HCC target for treatment is urgently required [6, 7].

Belongs to the aldo-keto reductase superfamily $[8,9]$, AKR1B10 is predominantly expressed in the small intestine, colon, liver, thymus and adrenal gland $[8,10]$. Upregulation of AKR1B10 has been observed in various human malignancies, including non-small cell lung carcinoma [11], pancreatic cancer [12], breast cancer [13, 14], oral squamous cell carcinoma [15], nasopharyngeal carcinoma [16] and HCC [17, 18]. 
Conversely, underexpression of AKR1B10 has been observed in colon [19], gastric [20], endometrial cancer [21] and head and neck cancers [22]. However, AKR1B10 in HCC has been controversially discussed. Some researches revealed that negatively correlated with serum alpha-fetoprotein (AFP) level, high AKR1B10 expression was found to be a favorable factor of overall survival and disease-free survival in patients with hepatitis B virus-related HCC [23-25]. Another study found that high AKR1B10 expression was an independent risk factor for HCC development [26] and significantly correlated with liver damage[25]. Thus, to reevaluate the prognostic value of AKR1B10 in HCC patients is of great importance.

To help elucidate the possible relationship between AKR1B10 expression and HCC outcomes, we identified the AKR1B10 expression in GEO, Oncomine and TCGA databases and performed a survival analysis based on TCGA profile, in the hope of providing useful insights into hepatocarcinogenesis and aggressiveness.

\section{Materials and Methods}

\section{Data resource and Description}

Seven expression microarray series GSE14520, GSE25097, GSE33006, GSE45436, GSE55092, GSE60502 and GSE77314 containing HCC tumor and nontumor samples were downloaded from the Gene Expression Omnibus dataset (GEO, https://www.ncbi.nlm.nih.gov/geo/). Platforms and samples of GEO series were summarized in Table 1.

TCGA liver hepatocellular carcinoma mRNA normalized counts data derived from RNAseq Htseq platform were downloaded from Genomic Data Commons Data Portal (https://cancergenome.nih .gov/). TCGA RNAseq data contains 424 samples with 374 primary tumor and 50 solid tissue normal samples. RTCGAToolbox [27] and edgeR [28, 29] packages were used to identify AKR1B10 expression between tumor and normal samples.

\section{Bioinformatics analysis for identifying AKR 1 B 10 expression}

Raw.CEL files of the microarray from each GEO dataset were normalized by quantile method of Robust Multichip Analysis (RMA) from $R$ affy package [30]. Gene expression of AKR1B10 was performed by tumor and normal comparison from $\mathrm{R}$ Limma package [31]. Gene expression data of GSE77314 in XLSX form was downloaded from GEO dataset directly.

Studies compared AKR1B10 between cancer and normal samples in liver cancer were selected with threshold by $\mathrm{p}$-Value $\leq 1 \mathrm{E}-4$, fold change $\geq 2$ and top
$10 \%$ gene rank in Oncomine database (https://www.oncomine.org/).

\section{Survival analysis}

Liver Hepatocellular Carcinoma (TCGA, Provisional) database in cBioPortal for cancer genomics web service was used for identifying AKR1B10 for predicting the overall survival (OS) of HCC patients [32, 33]. AKR1B10 Mrna expression levels calculated by $\log 2$ calculation were compared based on clinical attribute in HCC patients. To evaluate associations between candidate biomarkers and survival and 4893 linic-pathological features in HCC patients, gene data with $\mathrm{Z}$ scores and clinical data of HCC patients in Liver Hepatocellular Carcinoma (TCGA, Provisional) database were downloaded from cBioPortal and matched using VLOOKUP index in EXCEL. After excluding ten patients with liver histology of hepatocholangiocarcinoma $(n=7)$ and fibrolamellar carcinoma $(\mathrm{n}=3)$ and six patients without gene expression levels, 361 HCC patients were included in the analysis. Additionally, the Kaplan Meier plotter online service (http://kmplot.com/analysis/) [34] was used for subgroup survival analysis of AKR1B10 with median cutoff in HCC patients.

\section{Protein-protein interaction and pathway/biological process enrichment}

Protein-Protein Interaction analysis (PPI) for AKR1B10 were performed by STRING database (https://string-db.org/). KEGG pathway and GO biological process enrichment analysis were conducted using Gene Set Enrichment Analysis (GSEA, http://software.broadinstitute.org/gsea/ index.jsp). To investigate gene sets, AKR1B10 interactive genes were uploaded to Molecular Signatures Database in GSEA. A false discovery rate $P$ value cut-off of $<0.05$ was set as the screening condition.

\section{Statistical analysis}

Differences of gene expression between the individual groups were analyzed using student $t$ test, Mann-Whitney $U$-test, Chi-square test and Ridit analysis based on variables types. PASW Statistics software version 23.0 from SPSS Inc. (Chicago, IL, USA) was used. A two-tailed $P<0.05$ were considered significant for all tests.

\section{Results}

\section{AKR IB 10 expression comparison}

The details of GEO series included in this analysis were summarized in Table 1 . As shown in Figure 1, AKR1B10 Mrna was significantly 
overexpressed in GSE14520, GSE25097, GSE33006, GSE45436, GSE55092, GSE60502. GSE77314 and TCGA datasets (all $P<0.01$, Figure 1 ).

For validation, we performed meta-analysis of AKR1B10 expression in 7 analyses with threshold by p-Value $\leq 1 \mathrm{E}-4$, fold change $\geq 2$ and top $10 \%$ gene rank in Oncomine database. As shown in Figure 2, compared with that in normal liver tissues, AKR1B10 was significantly upregulated in cirrhosis $(P<0.0001$, Figure 2A, 2B and 2E), liver cell dysplasia $(P<0.0001$, Figure $2 \mathrm{~A}$ and $2 \mathrm{E})$ and HCC tumors $(P<0.0001$, Figure 2A - 2E). Additionally, AKR1B10 was significantly higher in HCC tumor tissues than that in cirrhosis $(P<0.01$, Figure $2 \mathrm{~B}$ and $2 \mathrm{E})$ and in liver cell dysplasia $(P<0.05$, Figure $2 \mathrm{E})$.

\section{Associations between AKR1B10 and OS in HCC patients}

Using hash and survival packages in R program, we performed Kaplan-Meier analysis of AKR1B10 and OS in HCC patients, which showed that AKR1B10 overexpression in tumor tissues was significantly associated with poor OS in HCC patients ( $\log$ rank $P=0.004$, Figure 3A). A consistent result was validated in Kapan-Meier Plotter online service as shown in Figure 3B ( $\log$ rank $P=0.014$, Figure 3B). Moreover, subgroup analysis revealed that AKR1B10 upregulation in tumors was risk factor for 1-year, 3-year and 5-year OS in HCC patients $(\mathrm{HR}=1.74, \log$ $\operatorname{rank} P=0.041 ; \mathrm{HR}=1.51, \log \operatorname{rank} P=0.039$ and $\mathrm{HR}=$ $1.53, \log \operatorname{rank} P=0.021$, respectively, Figure $3 C-3 E$ ).

In addition, we performed subgroup survival analysis in different population. As shown in Figure 4, high AKR1B10 level significantly contributed to worse OS in HCC patients without hepatitis virus infection $(\mathrm{HR}=2.24, \log \operatorname{rank} P=0.00055$, Figure $4 \mathrm{~B})$. And, AKR1B10 overexpression was significantly associated with OS both in male and female $(\mathrm{HR}=$ 1.77, $\log$ rank $P=0.014$ and $H R=1.86, \log$ rank $P=$ 0.034, respectively, Figure 4C and 4D). AKR1B10 was risk factor for OS in White HCC ( $\mathrm{HR}=2.04, \log$ rank $P$ $=0.0029$, Figure 4E) and in cases with alcohol consumption $(\mathrm{HR}=2.28, \log$ rank $P=0.013$, Figure $4 G)$.

In HCC patients with neoplasm histologic Grade III, AKR1B10 was significantly associated with poor OS $(\mathrm{HR}=2.04, \log$ rank $P=0.023$, Figure 5C), while no differences were found in HCC cases with Grade I and Grade II ( $\log$ rank $P>0.05$, Figure 5A and 5B). Moreover, AKR1B10 overexpression was significantly contributed to poor OS in HCC patients with stage II-III $(\mathrm{HR}=1.83, \log$ rank $P=0.013$, Figure 5E) and stage III-IV $(\mathrm{HR}=1.98, \log$ rank $P=0.021$, Figure 5F).

Table 1. Details of GEO series included in this analysis

\begin{tabular}{lllll}
\hline GEO series & Contributor(s) & Tumor & Nontumor & Platform \\
\hline GSE14520 & Roessler S et al, 2009 & 222 & 212 & Affymetrix Human Genome U133A 2.0 Array / Affymetrix HT Human Genome U133A Array \\
GSE25097 & Zhang C, 2010 & 268 & 243 & Rosetta/Merck Human RSTA Affymetrix 1.0 microarray, Custom CDF \\
GSE33006 & Huang Y et al, 2011 & 3 & 3 & Affymetrix Human Genome U133 Plus 2.0 Array \\
GSE45436 & Hsieh J, 2013 & 97 & 37 & Affymetrix Human Genome U133 Plus 2.0 Array \\
GSE55092 & Melis M et al, 2014 & 49 & 91 & Affymetrix Human Genome U133 Plus 2.0 Array \\
GSE60502 & Kao KJ, 2014 & 18 & 18 & Affymetrix Human Genome U133A Array \\
GSE77314 & Hou G et al, 2016 & 50 & 50 & Illumina Genome Analyzer (Homo sapiens) \\
\hline
\end{tabular}
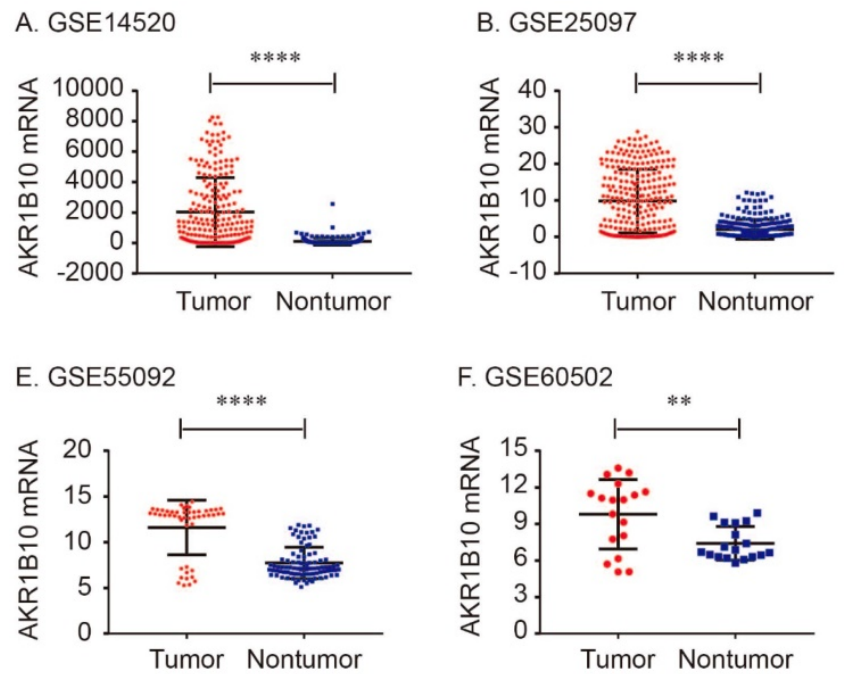
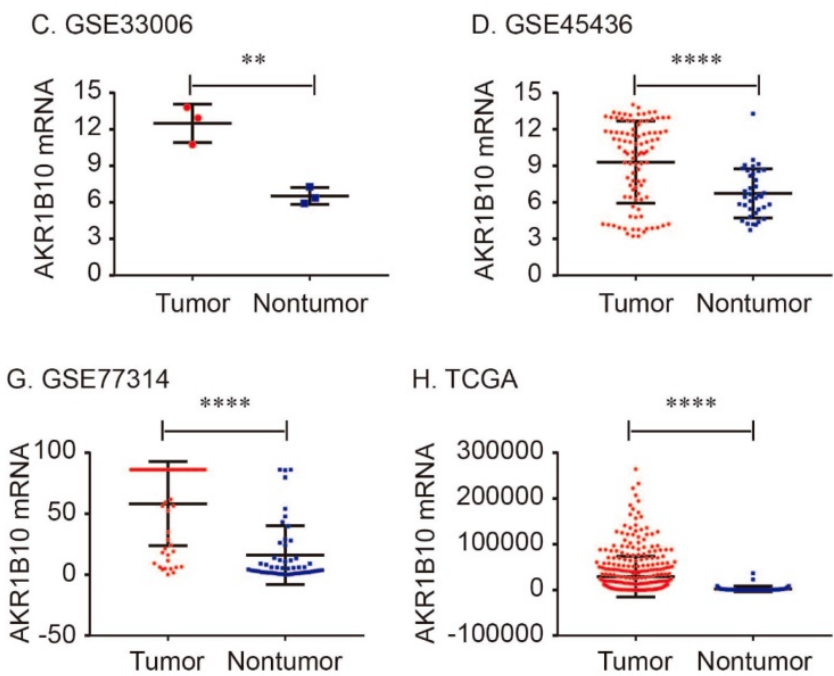

Figure 1. AKR1B10 mRNA expression levels between tumor and nontumor tissues in HCC patients in GEO database series including GSE14520 (A), GSE25097 (B), GSE33006 (C), GSE45436 (D), GSE55092 (E), GSE60502 (F), GSE77314 (G) and TCGA database (H). 
A. Meta-analysis of AKR1B10 in 7 Analyses

Threshold by:

- p-Value: $1 \mathrm{E}-4$

- Fold change: 2

- Gene rank: Top $10 \%$

\begin{tabular}{|c|c|c|}
\hline Median Rank & p-Value & Gene \\
\hline 382.0 & $3.84 \mathrm{E}-5$ & AKR1B10 \\
\hline
\end{tabular}

Legend

1. Cirrhosis vs. Normal, Mas Liver, Mol Med, 2008

2. Hepatocellular Carcinoma vs. Normal, Mas Liver, Mol Med, 2008

3. Hepatocellular Carcinoma vs. Normal, Roessler Liver, Cancer Res, 2010

4. Hepatocellular Carcinoma vs. Normal, Roessler Liver 2, Cancer Res, 2010

5. Cirrhosis vs. Normal, Wurmbach Liver, Hepatology, 2007

6. Hepatocellular Carcinoma vs. Normal, Wurmbach Liver, Hepatology, 2007

7. Liver Cell Dysplasia vs. Normal, Wurmbach Liver, Hepatology, 2007

$151025 \quad 251051$

$\underset{\square}{\square} \square \square \square \square$ Not measured

The rank for a gene is the median rank for that gene across each of the analyses.

The $\mathrm{p}$-Value for a gene is its $\mathrm{p}$-Value for the median-ranked analysis.

B. Mas Liver

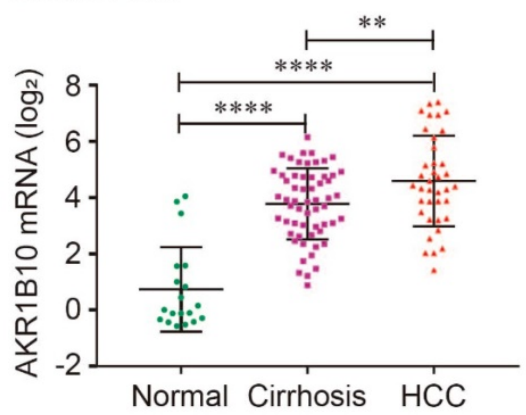

D. Roessler Liver 2

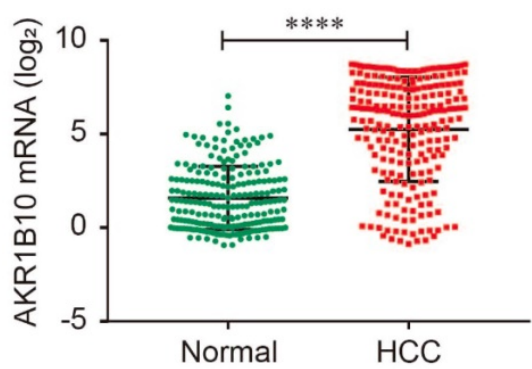

C. Roessler Liver
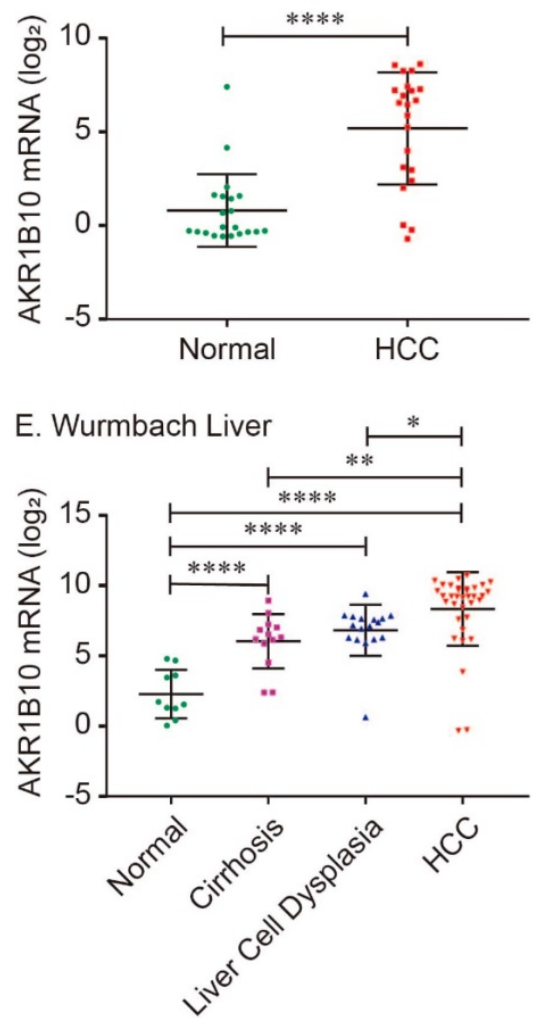

Figure 2. Comparison of AKR1B10 mRNA expression levels across 7 analyses in Oncomine database. Meta-analysis of AKR1B10 expression in 7 analyses (A); AKR1B10 levels in normal, cirrhosis and HCC tissues in Mas Liver (B); AKR1B10 levels in normal and HCC tissues in Roessler Liver (C) and Roessler Liver 2 (D); AKR1B10 levels in normal, cirrhosis, liver cell dysplasia and HCC tissues in Wurmbach Liver (E).

\section{Association between AKR1B 10 and clinico-pathological features in HCC patients}

As shown in Table 2, more male cases in AKR1B10 high group $(76.2 \%$ vs. $58.9 \%, P<0.001)$ and patients in AKR1B10 high group were significantly older than those in AKR1B10 low group (62.0yr vs. $59.5 y r, P=0.031$ ). More HCC cases had family history of cancer in AKR1B10 high group than those in AKR1B10 low group ( $43.1 \%$ vs. $30.0 \%, P=0.01)$. More 
HCC cases had hepatocarcinoma risk factors (especially alcohol consumption) in AKR1B10 high group than those in AKR1B10 low group $(P=0.006)$. As we expected, HCC patients in AKR1B10 high group suffered from significantly advanced Ishak fibrosis status and advanced hepatic inflammation $(P$ $=0.036$ and $P=0.033$, respectively) .

\section{PPI and KEGG/GO biological process enrichment}

PPI analysis using STRING revealed that 10 genes including DCXR, ALDH9A1, ALDH7A1, ENSG00000257767, LCT, GALM, SORD, ALDH1B1, $\mathrm{DHDH}$ and ALDH3A2 were interacted with AKR1B10 (Figure 6A). KEGG pathway enrichment of AKR1B10 interactive genes showed that metabolic

(A)

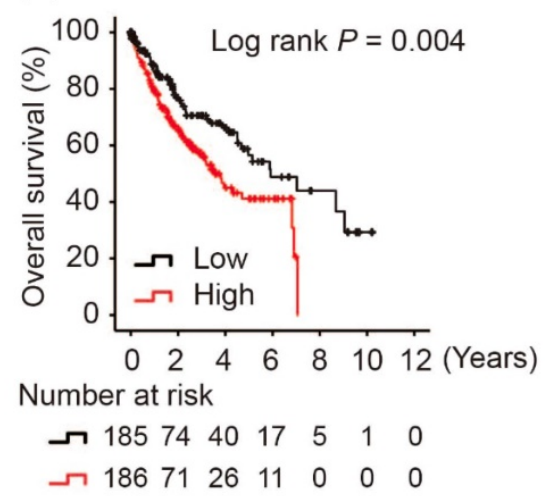

(C)
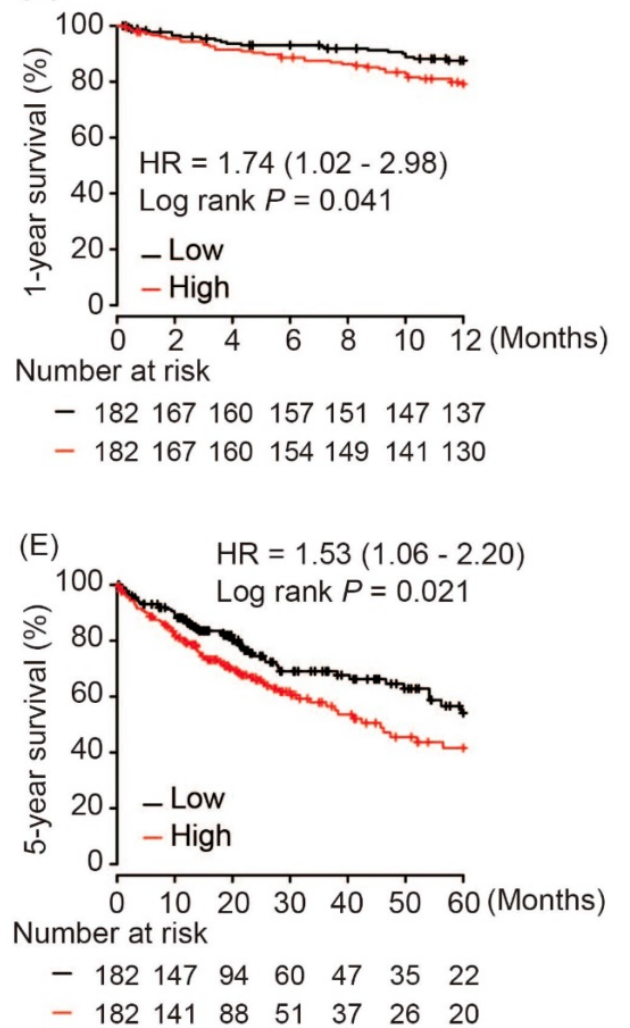

pathways, pentose and glucuronate interconversions, pyruvate metabolism, glycerolipid metabolism, glycolysis / gluconeogenesis, ascorbate and aldarate metabolism, histidine metabolism, beta-Alanine metabolism, propanoate metabolism, tryptophan metabolism, arginine and proline metabolism, fatty acid degradation, etc were the most enriched pathways (Figure 6B). Additionally, oxidation-reduction process, metabolic process including small molecular, organic hydroxy compound, alcohol, carbohydrate, single-organism, cellular aldehyde, glucose, ammonium ion, etc were most enriched GO biological process of AKR1B10 interactive genes (Figure 6B).

(B)

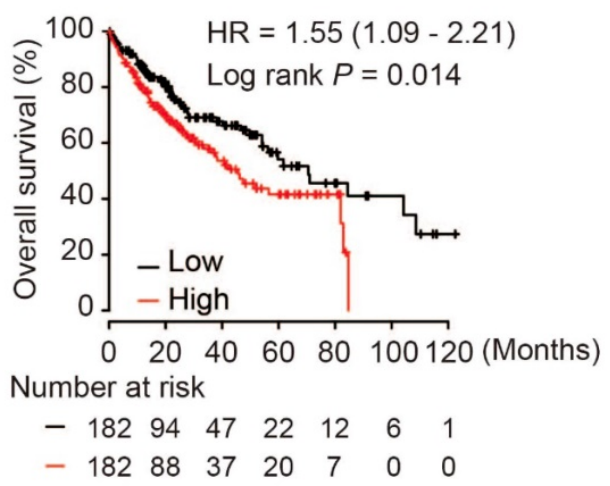

(D)

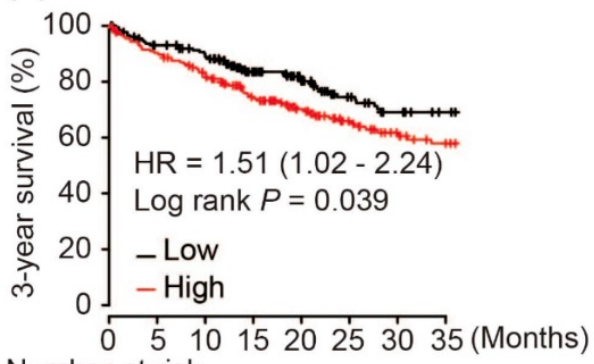

Number at risk

- 18215714711394716055

- 18215814111088675143

Figure 3. Overall survival of HCC patients grouped by AKR1B10 median cutoff in TCGA database (A, B); 1-year (C), 3-year (D) and 5-year overall survival comparison between AKR1B10 high and low groups. 
A. Hepatitis virus: yes

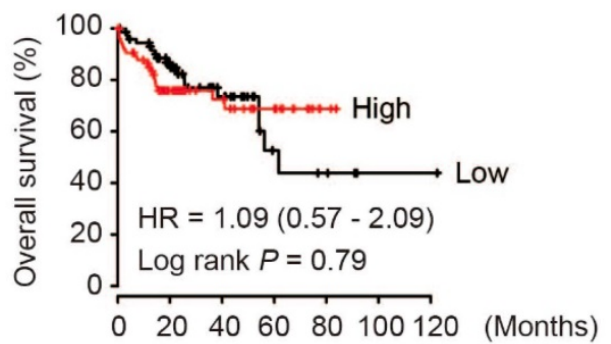

Number at risk

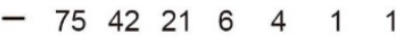

$$
\begin{aligned}
& \text { - } 75372113200
\end{aligned}
$$

\section{Male}

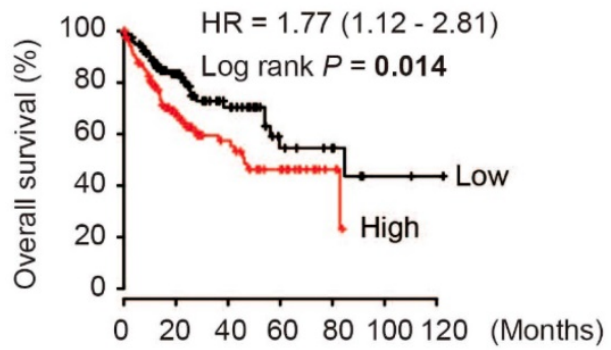

Number at risk

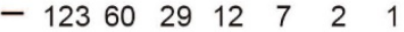

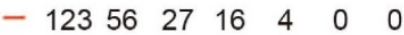

E. White

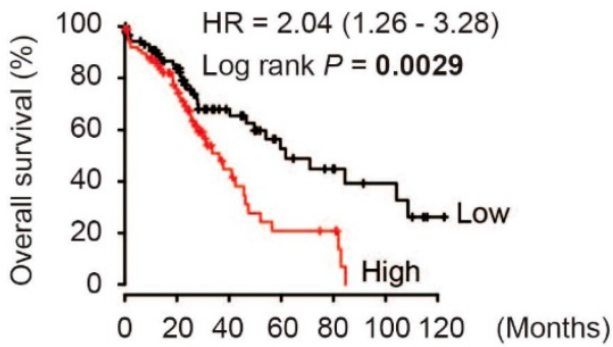

Number at risk

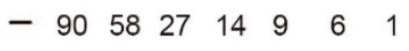

-914814650
0

G. Alcohol consumption: yes

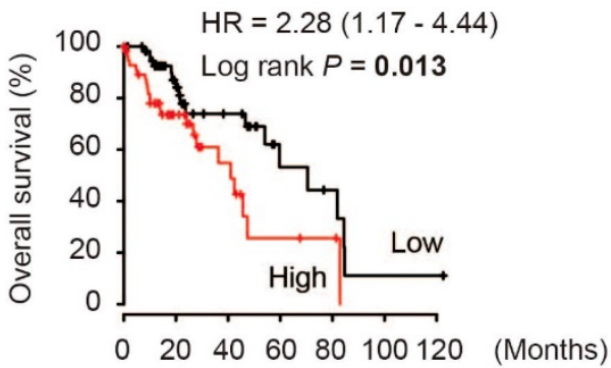

Number at risk

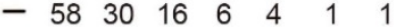

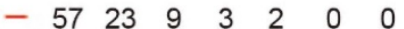

B. Hepatitis virus: none

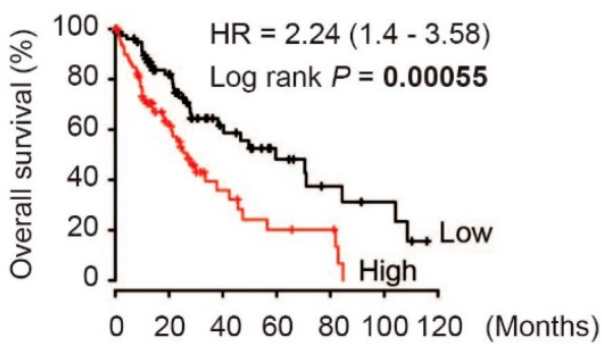

Number at risk

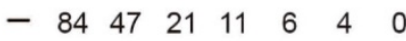

$$
\begin{aligned}
& \text { - } 8332105400
\end{aligned}
$$

D. Female

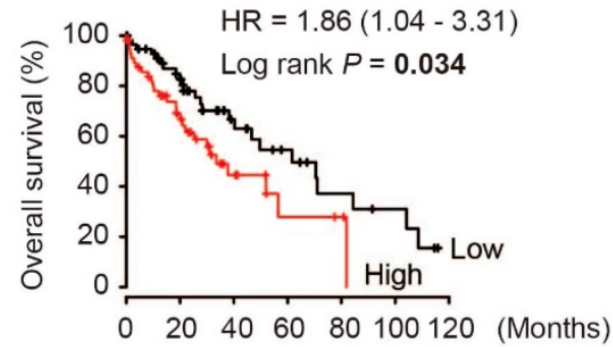

Number at risk

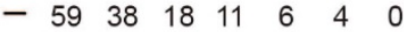

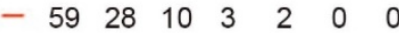

F. Asian

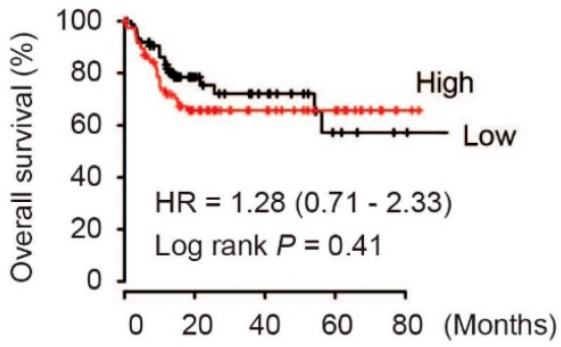

Number at risk

$\begin{array}{ccccc}-78 & 28 & 17 & 6 & 3 \\ -77 & 38 & 23 & 14 & 2\end{array}$

H. Alcohol consumption: no

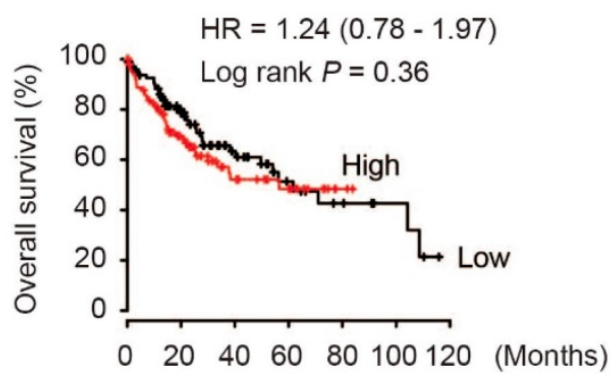

Number at risk

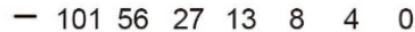

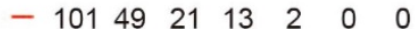

Figure 4. Subgroup analyses of overall survival comparison in different population [hepatitis virus infection status (A, B), gender (C, D), race (E, F) and alcohol consumption (G, H)] with AKR1B10 median cutoffs in HCC patients. 


\section{A. Grade I}

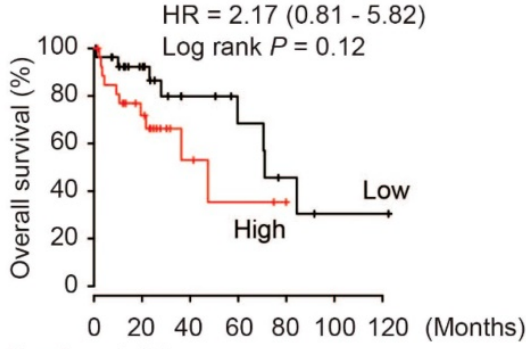

Number at risk

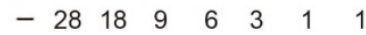

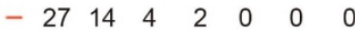

D. Stage I - II

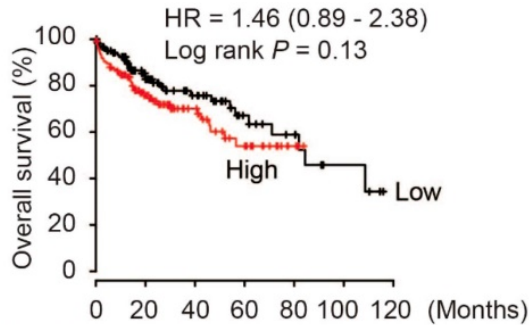

Number at risk

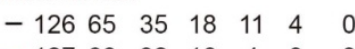

B. Grade II

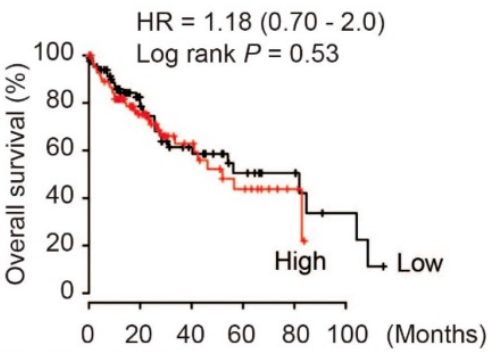

Number at risk

$\begin{array}{lllll}-87 & 41 & 22 & 12 & 8\end{array}$

$\begin{array}{llllll}-87 & 42 & 19 & 10 & 3 & 0\end{array}$

E. Stage II - III

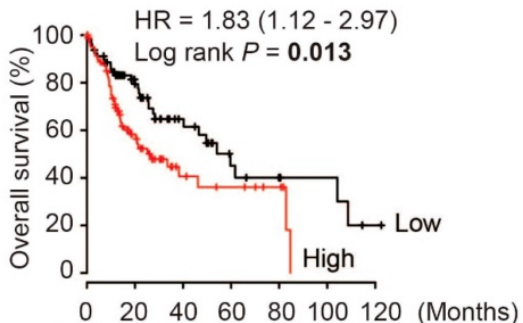

Number at risk

$\begin{array}{lllllll}-83 & 43 & 20 & 9 & 6 & 4 & 1\end{array}$
C. Grade III

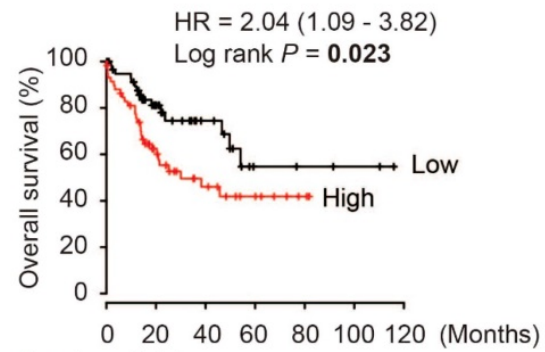

Number at risk

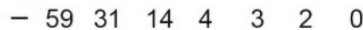

$\begin{array}{llllll}- & 5927 & 13 & 7 & 2 & 0\end{array}$

F. Stage III - IV

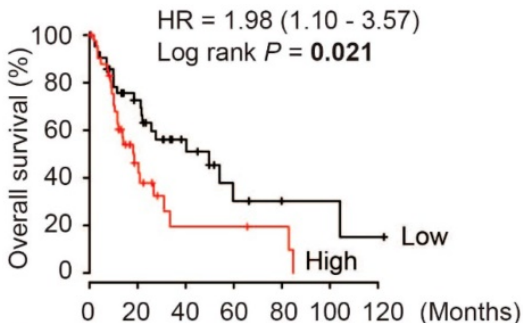

Number at risk

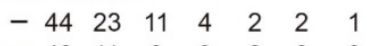

Figure 5. Overall survival of HCC patients grouped by AKR1B10 median in different grades $(A, B, C)$ and stages (D, E, F).

Table 2. Characteristics of HCC patients between AKR $1 \mathrm{~B} 10$ high and low groups

\begin{tabular}{|c|c|c|c|}
\hline \multirow[t]{2}{*}{ Variables } & \multicolumn{2}{|c|}{ AKR1B10 expression level } & \multirow[t]{2}{*}{$P$ value } \\
\hline & Low $(n=180)$ & High $(n=181)$ & \\
\hline Gender, male (\%) & $106(58.9)$ & $138(76.2)$ & $<0.001$ \\
\hline Age, median (IQR), years & $59.5(20)$ & $62(15)$ & 0.031 \\
\hline BMI, kg/m², n (\%) & & & 0.132 \\
\hline$<18.5$ & $14(7.8)$ & $7(3.9)$ & \\
\hline $18.5 \sim 24.99$ & $79(43.9)$ & $73(40.3)$ & \\
\hline $25 \sim 29.99$ & $36(20.0)$ & $52(28.9)$ & \\
\hline$>30$ & $35(19.4)$ & $32(17.7)$ & \\
\hline NA & $16(8.9)$ & $17(9.4)$ & \\
\hline Race, n (\%) & & & 0.36 \\
\hline Asian & $77(42.8)$ & 79 (43.6) & \\
\hline White & $91(50.6)$ & $85(47.0)$ & \\
\hline Black or African American & $7(3.9)$ & $10(5.5)$ & \\
\hline American Indian or Alaska Native & $2(1.1)$ & $0(0)$ & \\
\hline NA & $3(1.6)$ & $7(3.9)$ & \\
\hline Tumor status, n (\%) & & & 0.923 \\
\hline With tumor & $53(29.4)$ & $56(30.9)$ & \\
\hline Tumor free & $115(63.9)$ & $112(61.9)$ & \\
\hline NA & $12(6.7)$ & $13(7.2)$ & \\
\hline Family history of cancer, n (\%) & $54(30.0)$ & $78(43.1)$ & 0.01 \\
\hline $\begin{array}{l}\text { Hepatocarcinoma risk factors*, n } \\
(\%)\end{array}$ & & & 0.006 \\
\hline Hepatitis virus infection & $55(30.6)$ & $56(30.9)$ & \\
\hline Alcohol consumption & $48(26.7)$ & $67(37.0)$ & \\
\hline Non-alcoholic fatty liver disease & $6(3.3)$ & $6(3.3)$ & \\
\hline No risk factors & $54(30.0)$ & $32(17.7)$ & \\
\hline Other & $8(4.4)$ & $10(5.5)$ & \\
\hline NA & $9(5.0)$ & $10(5.5)$ & \\
\hline Neoplasm histologic grade, $\mathrm{n}(\%)$ & & & 0.054 \\
\hline G1 & $35(19.4)$ & $18(9.9)$ & \\
\hline G2 & $76(42.2)$ & $95(52.5)$ & \\
\hline G3 & $60(33.3)$ & $61(33.7)$ & \\
\hline G4 & $6(3.3)$ & $5(2.8)$ & \\
\hline NA & $3(1.7)$ & $2(1.1)$ & \\
\hline
\end{tabular}

\begin{tabular}{|c|c|c|c|}
\hline \multirow[t]{2}{*}{ Variables } & \multicolumn{2}{|c|}{ AKR1B10 expression level } & \multirow[t]{2}{*}{$P$ value } \\
\hline & Low $(n=180)$ & $\operatorname{High}(\mathrm{n}=181)$ & \\
\hline AJCC stage, n (\%) & & & 0.203 \\
\hline I & $84(46.7)$ & $83(45.9)$ & \\
\hline II & $35(19.4)$ & $47(26.0)$ & \\
\hline III & $48(26.7)$ & $33(18.2)$ & \\
\hline IV & $4(2.2)$ & $3(1.7)$ & \\
\hline NA & $9(5.0)$ & $15(8.3)$ & \\
\hline Vascular invasion, $\mathrm{n}(\%)$ & & & 0.999 \\
\hline Macro & $8(4.4)$ & $8(4.4)$ & \\
\hline Micro & $44(24.4)$ & $45(24.9)$ & \\
\hline None & $99(55.0)$ & $101(55.8)$ & \\
\hline NA & $29(16.1)$ & $27(14.9)$ & \\
\hline Child-pugh classification, n (\%) & & & 0.525 \\
\hline $\mathrm{A}$ & $101(56.1)$ & $111(61.3)$ & \\
\hline B & $9(5.0)$ & $12(6.6)$ & \\
\hline $\mathrm{C}$ & $1(0.6)$ & $0(0)$ & \\
\hline NA & $69(38.3)$ & $58(32.0)$ & \\
\hline $\mathrm{AFP}>400 \mathrm{ng} / \mathrm{ml}, \mathrm{n}(\%)$ & 39 (21.7) & $25(13.8)$ & 0.051 \\
\hline Platelet, median (IQR), $\times 10^{3} / \mathrm{mm}^{3}$ & $204.5(182)$ & $178(136)$ & 0.066 \\
\hline $\begin{array}{l}\text { New tumor event after initial } \\
\text { treatment, } \mathrm{n}(\%)\end{array}$ & $46(25.6)$ & $47(26.0)$ & 0.929 \\
\hline Ishak fibrosis status, $\mathrm{n}(\%)$ & & & 0.036 \\
\hline No fibrosis & $44(24.4)$ & $28(15.5)$ & \\
\hline Portal fibrosis & $10(5.6)$ & 21 (11.6) & \\
\hline Fibrous speta & $11(6.1)$ & $17(9.4)$ & \\
\hline $\begin{array}{l}\text { Nodular Formation/Incomplete } \\
\text { Cirrhosis }\end{array}$ & $3(1.7)$ & $6(3.3)$ & \\
\hline Cirrhosis & $30(16.7)$ & $40(22.1)$ & \\
\hline NA & $82(45.6)$ & $69(38.1)$ & \\
\hline Hepatic inflammation, $\mathrm{n}(\%)$ & & & 0.033 \\
\hline None & 69 (38.3) & $45(24.9)$ & \\
\hline Mild & $43(23.9)$ & $54(29.8)$ & \\
\hline Severe & $7(3.9)$ & $11(6.1)$ & \\
\hline NA & $61(33.9)$ & $71(39.2)$ & \\
\hline Follow up, median (IQR), years & $0.378(1.8)$ & $0.334(1.3)$ & 0.453 \\
\hline
\end{tabular}

IQR, interquartile range; BMI, body mass index; AJCC, American Joint Committee on Cancer; AFP, alpha-fetoprotein; NA, not available.

* Sum of all risk factors compared with no risk factors. 
A. Protein-protein interaction of AKR1B10 in String

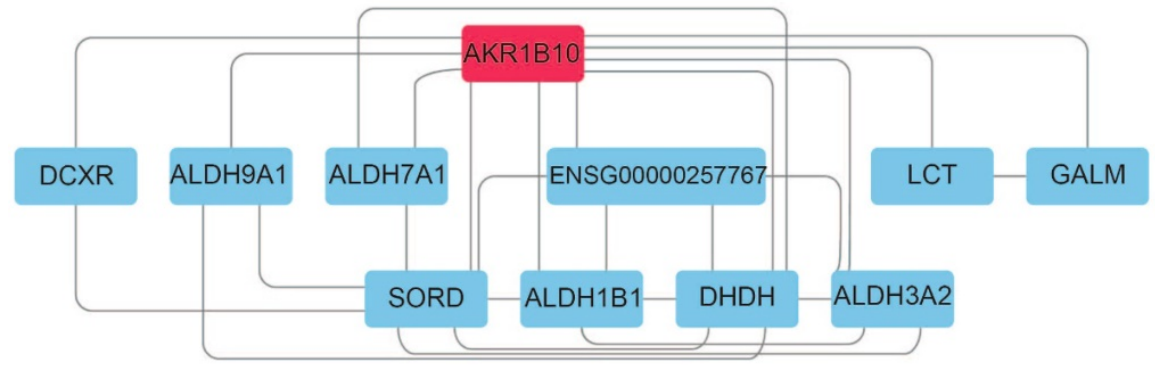

B. KEGG and GO biological process enrichment of AKR1B10 interactive genes

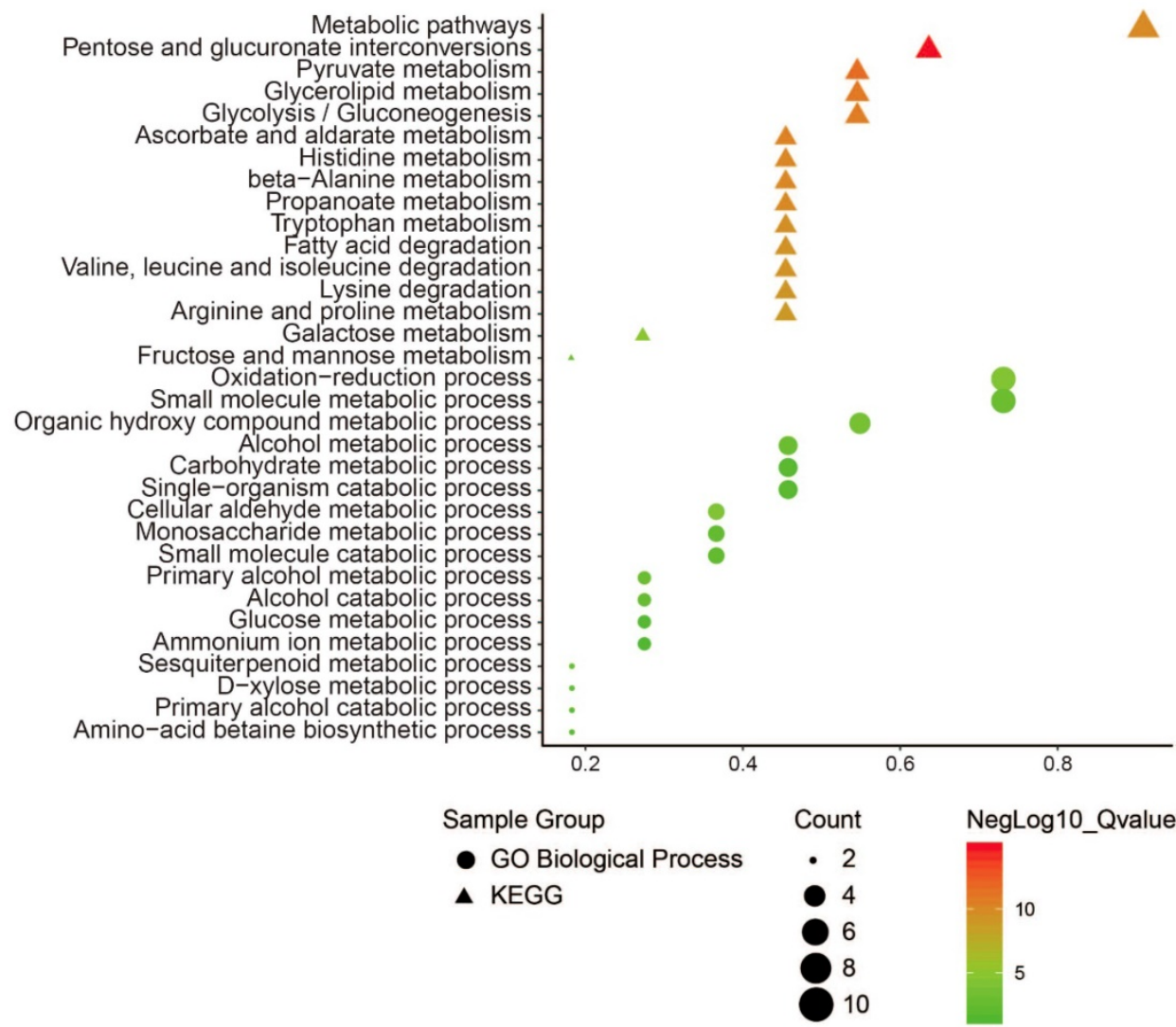

Figure 6. Protein-protein interaction of AKR1B10 using String analysis (A); KEGG and GO biological process enrichment of its interactive genes (B)

\section{Discussion}

Over the past few years, new data have recently emerged that implicate AKR1B10 in tumor carcinogenesis in different systemic malignancies [35]. AKR1B10 is expressed at lower levels in normal liver $[8,10]$ and overexpressed in HCC tumors [22, 25]. Matkowskyj $\mathrm{K}$ et al reported that AKR1B10 was overexpressed in $97 \%$ of HCC, with minimal to no expression in adjacent hepatic tissue, while hepatic adenomas and focal nodular hyperplasia did not exhibit expression of AKR1B10 [17]. A small sample study demonstrated that increased expression of AKR1B10 in moderately-differentiated HCC compared with well-differentiated HCC, poorly-differentiated HCC, and liver cirrhosis [18].
Consistent with previous reports, we found that AKR1B10 was significantly upregulated in cirrhosis, liver cell dysplasia and HCC tumors compared with normal livers. Additionally, AKR1B10 was significantly higher in HCC tumor tissues than that in cirrhosis and in liver cell dysplasia. Hence, we assumed that AKR1B10 is emerging as a biomarker to distinguish hepatocellular carcinoma from benign liver lesions.

However, roles of AKR1B10 in hepatocarcinogenesis are still controversial. In a retrospective study of 168 cases, Schmitz $\mathrm{K}$ et al found that loss of AKR1B10 expression correlates with increased proliferative activity. A poorer prognosis in AKR1B10-negative HCCs was revealed compared with patients with strongly positive HCCs [36]. In 110 
patients with hepatitis B virus-related HCC, high AKR1B10 expression was negatively correlated with serum AFP level. Patients with high AKR1B10 expression had significantly higher DFS than those with low expression within 2 years after liver resection. High AKR1B10 expression was found to be a favorable factor of early recurrence and OS in HCC patients [23]. In 255 HCC patients who underwent curative hepatectomy, high AKR1B10 expression was significantly associated with a lack of invasion of the major portal vein, a lack of intrahepatic metastasis, lower tumor stages, and lower AFP levels, which was found to be an independent predictor of both longer recurrence-free survival and longer disease-specific survival [24].

Conversely, our results demonstrated that high AKR1B10 in tumors were significantly associated with worse OS in HCC patients. AKR1B10 overexpression were associated with poor 1-year, 3-year and 5-year OS. A series publication enhanced our findings. Murata A et al reported that high AKR1B10 expression was an independent risk factor for HCC development in chronic hepatitis $C$ patients. During the follow-up period after viral eradication, patients expressing high levels of AKR1B10 expressed markedly higher levels of alanine aminotransferase and AFP than did patients exhibiting low AKR1B10 expression [26]. The oncogenetic functions of AKR1B10 in HCC tumor growth were validated in HCC cell lines SMMC-7721, HepG2 and Hep3B. Knockdown of AKR1B10 through shRNA in Hep3B cells showed significantly induced cell cycle arrest and inhibited cell growth [37]. ShRNA-mediated silencing of AKR1B10 expression in HCC cells resulted in increased cell apoptosis, decreased colony formation and size, and enhanced cytoreductive response following exposure to doxorubicin chemotherapy [17]. A recent study identified that AKR1B10 is a novel downstream target of interleukin-1 receptor-associated kinase 1 (IRAK1), which was found to be overexpressed in HCC and significantly correlated with IRAK1 expression. Knockdown of AKR1B10 negated IRAK1-induced tumor-initiating cells functions [38].

Functionally, AKR1B10 exerts a protective role through eliminating oxidative and carbonyl stresses and promoting epithelial proliferation for damage repair in inflammation. The KEGG pathway and GO biological process of AKR1B10 interactive genes in our study revealed that AKR1B10 should develop metabolic pathways and oxidation-reduction process. Unfortunately, we could not perform experimental research for probing potential oncogenic mechanisms of AKR1B10 in HCC development. And, no our own follow-up data of HCC patients were available.
Considered the current controversial publications, we suggest a meta-analysis to evaluate the links between AKR1B10 and HCC prognosis. Even though, considered previous reports, we cautiously drew the hypothesis that AKR1B10 overexpression contributed to unfavorable prognosis in HCC patients.

\section{Acknowledgements}

This work was supported by National Natural Science Foundation of China (grant number 81803901 and 31601908) and Shanghai Sailing Program (grant number 17YF1416000).

\section{Competing Interests}

The authors have declared that no competing interest exists.

\section{References}

1. Bray F, Ferlay J, Soerjomataram I, et al. Global Cancer Statistics 2018: GLOBOCAN Estimates of Incidence and Mortality Worldwide for 36 Cancers in 185 Countries. CA Cancer J Clin. 2018; 68: 394-424.

2. Heimbach JK, Kulik LM, Finn RS, et al. AASLD guidelines for the treatment of hepatocellular carcinoma. Hepatology. 2018; 67: 358-80.

3. Omata M, Cheng AL, Kokudo N, et al. Asia-Pacific clinical practice guidelines on the management of hepatocellular carcinoma: a 2017 update. Hepatol Int. 2017; 11: 317-70.

4. Siegel RL, Miller KD, Jemal A. Cancer Statistics, 2017. CA Cancer J Clin. 2017; 67: 7-30

5. Petrick JL, Kelly SP, Altekruse SF, et al. Future of Hepatocellular Carcinoma Incidence in the United States Forecast Through 2030. J Clin Oncol. 2016; 34: 1787-94.

6. Guo W, Tan HY, Wang N, et al. Deciphering hepatocellular carcinoma through metabolomics: from biomarker discovery to therapy evaluation. Cancer Manag Res. 2018; 10: 715-34.

7. Zhang B, Finn RS. Personalized Clinical Trials in Hepatocellular Carcinoma Based on Biomarker Selection. Liver Cancer. 2016; 5: 221-32.

8. Cao D, Fan ST, Chung SS. Identification and characterization of a novel human aldose reductase-like gene. J Biol Chem. 1998; 273: 11429-35.

9. Ma J, Cao D. Human aldo-keto reductases: structure, substrate specificity and roles in tumorigenesis. Biomol Concepts. 2011; 2: 115-26.

10. Hyndman DJ, Flynn TG. Sequence and expression levels in human tissues of a new member of the aldo-keto reductase family. Biochim Biophys Acta. 1998; 1399: 198-202.

11. Kang MW, Lee ES, Yoon SY, et al. AKR1B10 is associated with smoking and smoking-related non-small-cell lung cancer. J Int Med Res. 2011; 39: 78-85.

12. Chung YT, Matkowskyj KA, Li H, et al. Overexpression and oncogenic function of aldo-keto reductase family 1B10 (AKR1B10) in pancreatic carcinoma. Mod Pathol. 2012; 25: 758-66.

13. Reddy KA, Kumar PU, Srinivasulu M, et al. Overexpression and enhanced specific activity of aldoketo reductases (AKR1B1 \& AKR1B10) in human breast cancers. Breast. 2017; 31: 137-43.

14. Ma J, Luo DX, Huang C, et al. AKR1B10 overexpression in breast cancer: association with tumor size, lymph node metastasis and patient survival and its potential as a novel serum marker. Int J Cancer. 2012; 131: E862-71.

15. Ko HH, Peng $\mathrm{HH}$, Cheng SJ, et al. Increased salivary AKR1B10 level: association with progression and poor prognosis of oral squamous cell carcinoma. Head Neck. 2018; 40: 2642-7.

16. He YC, Shen Y, Cao Y, et al. Overexpression of AKR1B10 in nasopharyngeal carcinoma as a potential biomarker. Cancer Biomark. 2016; 16: 127-35.

17. Matkowskyj KA, Bai $\mathrm{H}$, Liao J, et al. Aldoketoreductase family 1B10 (AKR1B10) as a biomarker to distinguish hepatocellular carcinoma from benign liver lesions. Hum Pathol. 2014; 45: 834-43.

18. Han C, Gao L, Zhao L, et al. Immunohistochemistry Detects Increased Expression of Aldo-Keto Reductase Family 1 Member B10 (AKR1B10) in Early-Stage Hepatocellular Carcinoma. Med Sci Monit. 2018; 24: 7414-23.

19. Ohashi T, Idogawa M, Sasaki Y, et al. AKR1B10, a transcriptional target of p53, is downregulated in colorectal cancers associated with poor prognosis. Mol Cancer Res. 2013; 11: 1554-63.

20. Yao HB, Xu Y, Chen LG, et al. AKR1B10, a good prognostic indicator in gastric cancer. Eur J Surg Oncol. 2014; 40: 318-24.

21. Hevir N, Sinkovec J, Lanisnik Rizner T. Decreased levels of AKR1B1 and AKR1B10 in cancerous endometrium compared to adjacent non-cancerous tissue. Chem Biol Interact. 2013; 202: 226-33.

22. Laffin B, Petrash JM. Expression of the Aldo-Ketoreductases AKR1B1 and AKR1B10 in Human Cancers. Front Pharmacol. 2012; 3: 104. 
23. Wang YY, Qi LN, Zhong JH, et al. High expression of AKR1B10 predicts low risk of early tumor recurrence in patients with hepatitis B virus-related hepatocellular carcinoma. Sci Rep. 2017; 7: 42199.

24. Ha SY, Song DH, Lee JJ, et al. High expression of aldo-keto reductase $1 \mathrm{~B} 10$ is an independent predictor of favorable prognosis in patients with hepatocellular carcinoma. Gut Liver. 2014; 8: 648-54.

25. Sonohara F, Inokawa $Y$, Hishida M, et al. Prognostic significance of AKR1B10 gene expression in hepatocellular carcinoma and surrounding non-tumorous liver tissue. Oncol Lett. 2016; 12: 4821-8.

26. Murata A, Genda T, Ichida T, et al. Pretreatment AKR1B10 expression predicts the risk of hepatocellular carcinoma development after hepatitis $\mathrm{C}$ virus eradication. World J Gastroenterol. 2016; 22: 7569-78.

27. Samur MK. RTCGAToolbox: a new tool for exporting TCGA Firehose data. PLoS One. 2014; 9: e106397.

28. Nikolayeva O, Robinson MD. edgeR for differential RNA-seq and ChIP-seq analysis: an application to stem cell biology. Methods Mol Biol. 2014; 1150: 45-79.

29. Robinson MD, McCarthy DJ, Smyth GK. edgeR: a Bioconductor package for differential expression analysis of digital gene expression data. Bioinformatics. 2010; 2: 139-40.

30. Gautier L, Cope L, Bolstad BM, et al. affy--analysis of Affymetrix GeneChip data at the probe level. Bioinformatics. 2004; 20: 307-15.

31. Ritchie ME, Phipson B, Wu D, et al. limma powers differential expression analyses for RNA-sequencing and microarray studies. Nucleic Acids Res. 2015; 43: e47.

32. Cerami E, Gao J, Dogrusoz U, et al. The cBio cancer genomics portal: an open platform for exploring multidimensional cancer genomics data. Cancer Discov. 2012; 2: 401-4.

33. Gao J, Aksoy BA, Dogrusoz U, et al. Integrative analysis of complex cancer genomics and clinical profiles using the cBioPortal. Sci Signal. 2013; 6: pl1.

34. Menyhárt O, Nagy A, Győrffy B. Determining consistent prognostic biomarkers of overall survival and vascular invasion in hepatocellular carcinoma. R Soc Open Sci. 2018; 5: 181006.

35. Kapoor S. AKR1B10 and its emerging role in tumor carcinogenesis and as a cancer biomarker. Int J Cancer. 2013; 132: 495.

36. Schmitz KJ, Sotiropoulos GC, Baba HA, et al. AKR1B10 expression is associated with less aggressive hepatocellular carcinoma: a clinicopathological study of 168 cases. Liver Int. 2011; 31: 810-6.

37. Wang J, Zhou Y, Fei X, et al. Biostatistics mining associated method identifies AKR1B10 enhancing hepatocellular carcinoma cell growth and degenerated by miR-383-5p. Sci Rep. 2018; 8: 11094.

38. Cheng BY, Lau EY, Leung HW, et al. IRAK1 Augments Cancer Stemness and Drug Resistance via the AP-1/AKR1B10 Signaling Cascade in Hepatocellular Carcinoma. Cancer Res. 2018; 78: 2332-42. 